Historic, Archive Document

Do not assume content reflects current scientific knowledge, policies, or practices. 


\section{BALLED AND BURLAPPED EVERGREENS}

Balling and burlapping an evergreen is the best known method of handling the larger and more expensive evergreens. Our B \& B evergreens are all specimen trees, several times transplanted, and just the thing for an immediate showing on your lawn.

\begin{tabular}{|c|c|c|c|c|c|}
\hline & size & each & & size & each \\
\hline Savin Juniper . & $1-11 / 4 \mathrm{ft}$. & $\$ 2.50$ & Siberian Arbor Vitae .. & $-11 / 4 \mathrm{ft}$. & $\$ 2.50$ \\
\hline Am. Arbor Vitae $\ldots \ldots \ldots \ldots \ldots \ldots$ & $11 / 2-2 \mathrm{ft}$. & 1.75 & Retinospora Plumosa & $-11 / 2 \mathrm{ft}$. & 3.50 \\
\hline Hovey Arbor Vitae $\ldots \ldots \ldots \ldots \ldots$. & $1-1 \frac{1 / 2}{\mathrm{ft}}$. & 2.50 & White Spruce $\ldots \ldots \ldots \ldots \ldots \ldots$. & $-11 / 2 \mathrm{ft}$. & 2.00 \\
\hline Globe Arbor Vitae & $1-11 / 4 \mathrm{ft}$. & 2.00 & Norway Spruce .... & $-11 / 4 \mathrm{ft}$. & 1.00 \\
\hline Globe Arbor Vitae ....... & 1 foot & 1.50 & Norway Spruce $\ldots . . .$. & $-3 \mathrm{ft}$. & 1.75 \\
\hline Pyramidal Arbor Vitae ......... & $11 / 2-2$ ft. & 3.00 & Col. Blue Spruce ... & $-1 \frac{1}{2} \mathrm{ft}$. & 3.50 \\
\hline Pyramidal Arbor Vitae .......... & $1-1 \frac{1 / 2}{\mathrm{ft}}$. & 2.50 & Col. Blue Spruce ... & 1 foot & 2.50 \\
\hline Austrian Pine $\ldots \ldots \ldots \ldots \ldots \ldots \ldots$ & 1 foot & 1.75 & Scotch Pine .... & $1 \frac{1 / 2-2}{\mathrm{ft}}$. & 1.50 \\
\hline \multirow[t]{3}{*}{ Hemlock ..... } & $1-11 / 4 \mathrm{ft}$ & 2.50 & Balsam Fir & $\begin{array}{lll}1 & -1 / 4 & \mathrm{ft} .\end{array}$ & 2.00 \\
\hline & \multicolumn{3}{|c|}{ EVERGREEN SEEDLINGS } & & \\
\hline & & 100 & & & 100 \\
\hline Am. Arbor Vitae $\ldots \ldots \ldots \ldots \ldots$. & 4- 8 in. & $\$ 3.50$ & Jack Pine....... . & $3-5$ in. & $\$ 2.50$ \\
\hline Am. Arbor Vitae ... & 3- 6 in. & 2.00 & Scotch Pine $\ldots \ldots \ldots \ldots \ldots \ldots \ldots$ & 6-10 in. & \\
\hline Balsam Fir $\ldots \ldots \ldots \ldots \ldots \ldots \ldots \ldots$ & 4- 8 in. & 4.00 & Scotch Pine $\ldots \ldots \ldots \ldots \ldots \ldots \ldots$ & 4- 6 in. & 2.50 \\
\hline Norway Spruce $\ldots \ldots \ldots \ldots \ldots \ldots \ldots$ & 3- 5 in. & 2.00 & Austrian Pine $\ldots \ldots \ldots \ldots \ldots \ldots \ldots$ & 4- 6 in. & 4.00 \\
\hline European Larch .... & $6-10$ in. & 3.00 & Western Pine ......... & 3- 5 in. & 3.00 \\
\hline Oriental Arbor Vitae & 4- 6 in. & 4.50 & Col. Blue Spruce ...... & $3-5$ in. & 5.00 \\
\hline
\end{tabular}

\section{SHADE TREE SEEDLINGS}

$\$ 4.00 \quad$ Hard Sugar Maple $\ldots \ldots \ldots \ldots \ldots, 1 \frac{11 / 2-2 \mathrm{ft} .}{}$

DON'T UISS THIS OFFER-For only $\$ 10.00$ we will send the following seedlings postpaid to any postoffice in the United States. ACT NOW!

100 Sugar Maple................111/2 ft.

50 Scotch Pine ............... 6 in.
100 Am. Arbor Vitae ..............4-8 in.

50 Norway Spruce ............. 3-5 in. $10 \mathrm{Col}$. Blue Spruce ............. 3-5 in.

Our litte booklet "Care and Culture of Evergreens" included.

\section{ORNAMENTAL SHRUBS}

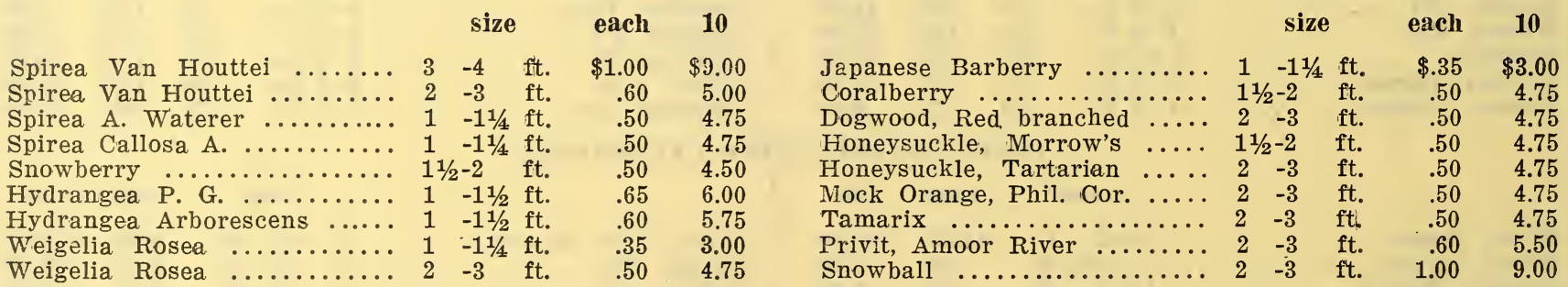

